San Jose State University

SJSU ScholarWorks

Faculty Publications

Psychology

January 2012

\title{
The effects of distractors in multiple object tracking are modulated by the similarity of distractor and target features
}

Cary S. Feria

San Jose State University, cary.feria@sjsu.edu

Follow this and additional works at: https://scholarworks.sjsu.edu/psych_pub

Part of the Psychology Commons

\section{Recommended Citation}

Cary S. Feria. "The effects of distractors in multiple object tracking are modulated by the similarity of distractor and target features" Perception (2012): 287-304. https://doi.org/10.1068/p7053

This Article is brought to you for free and open access by the Psychology at SJSU ScholarWorks. It has been accepted for inclusion in Faculty Publications by an authorized administrator of SJSU ScholarWorks. For more information, please contact scholarworks@sjsu.edu. 


\title{
The effects of distractors in multiple object tracking are modulated by the similarity of distractor and target features
}

\author{
Cary S Feria \\ Department of Psychology, One Washington Square, San Jose State University, San Jose, \\ CA 95192-0120, USA; e-mail: cary.feria@sjsu.edu \\ Received 23 June 2011, in revised form 5 March 2012
}

\begin{abstract}
Is the effect of distractors in multiple object tracking dependent on the distractors sharing the features of the targets? In experiment 1 , observers tracked five targets among five distractors that were identical to the targets and a number of additional distractors that were either identical to or featurally distinct from the targets. Results showed that distractors that are distinct from the targets in shape or color, or are stationary, impair tracking less than distractors that are identical to the targets. However, tracking performance declined as the number of distractors increased, even for featurally distinct distractors. Experiment 2 showed that distractors that differ from the targets on two features impair tracking less than distractors that differ from the targets on only one feature, but only when target tracking load is low. These results indicate that shape, color, and motion information about distractors can be used to distinguish them from targets during tracking, although even distractors with a different feature from the targets produce some interference with tracking. These findings suggest that the effect of distractors on tracking is dependent on top-down settings for target features.
\end{abstract}

\section{Introduction}

In daily life, the visual system must often track several target objects that are simultaneously moving through space among other distractor objects. This ability is demonstrated by soceer players tracking their team mates among opposing team members, parents tracking their children among others on a playground, and air traffic controllers tracking specified aircraft in a radar display. Often, the targets we are tracking have characteristics (such as their color, motion, or shape) that distinguish them from some of the other objects in the environment. However, studies on multiple object tracking (MOT - eg Alvarez and Franconeri 2007; Pylyshyn and Storm 1988) usually have utilized target and distractor objects that are identical, and thus little is known about how the characteristics of the distractors affect the ability to track the targets. The object of the current study was to find whether the similarity of the distractors to the targets affects the extent to which the distractors hinder tracking.

Several studies have demonstrated that target tracking ability is influenced by the presence of distractor objects. In variations of the MOT paradigm in which tracking could occur with or without distractors, tracking capacity is greater when no distractors are present (Horowitz and Cohen 2008; Horowitz et al 2007). Moreover, tracking performance declines as the number of distractors increases (Bettencourt and Somers 2009; Sears and Pylyshyn 2000).

Although the effects of the features of distractors on MOT ability has scarcely been investigated, many studies have been devoted to the effects of features of distractors on visual search and attention capture. During visual search, much research has indicated that the allocation of attention to a distractor object is affected by the distractor's physical salience (ie bottom - up processing - eg Franconeri et al 2005). Very salient stimuli, such as abrupt onset and uniquely colored objects, capture attention involuntarily (eg Theeuwes 1992, 1994). Yet much other research has indicated that the allocation of attention to a distractor object is affected by the distractor's relevance to current task demands (ie top-down processing - eg Folk et al 1992). Many studies have shown that the involuntary allocation of attention to a distractor object is dependent on whether the 
distractor shares features with the target (eg Folk et al 1992; Folk and Remington 1998). Visual search can be restricted to items that share the same color (or orientation, size, or motion direction) as the targets (eg Treisman and Sato 1990; Wolfe et al 1989). Treisman and Sato hold that this occurs because a preattentive parallel process filters distractors that do not share features with the target.

Why do distractors interfere with tracking the targets in MOT? A first explanation that has been proposed for distractor interference is that when a distractor passes near a target, the observer may mistake the distractor for the target (Alvarez and Franconeri 2007; Bettencourt and Somers 2009; Horowitz et al 2007; Intriligator and Cavanagh 2001; Iordanescu et al 2009; Oksama and Hyönä 2004; Pylyshyn 2004; Sears and Pylyshyn 2000). Additionally, some authors have suggested that the visual system attentionally suppresses distractors in order to reduce confusion with targets, and that this suppression consumes resources that could be used for tracking targets (Bettencourt and Somers 2009; Franconeri et al 2010; Horowitz et al 2007; Pylyshyn 2006; Shim et al 2008).

If the reason that distractors affect tracking is due to being confused with targets, then distractors that are less easily confusable with targets should interfere less with tracking. However, this prediction rests on the assumption that the objects' features are processed during MOT, and there has been contention in the literature about the extent to which this occurs. Several studies have suggested that little information about objects' features and identities is acquired (Pylyshyn 2004; Saiki 2003), or that information about features or identities is acquired for only a subset of the tracked targets (Botterill et al 2011; Cohen et al 2011; Fencsik et al 2007; Ren et al 2009). On the other hand, a number of other studies have suggested that some information about objects' features and identities indeed is acquired during tracking (Bahrami 2003; Oksama and Hyönä 2008; St Clair et al 2010; Yantis 1992). Makovski and Jiang (2009b) found that, when each object has a unique color, tracking performance is better than when all objects are identical. Similarly, Horowitz et al (2007) had observers track animal pictures and found that tracking performance was better when each object was a different animal picture than when all objects were the same animal picture.

A second reason that distractors may reduce tracking performance is because they are physically salient and divert attention away from tracking the targets (Bettencourt and Somers 2009). The salience of the distractors could be based on characteristics such as their luminance contrast and motion transients. Bettencourt and Somers also suggest that attentional suppression is used to prevent the distractors from drawing attention in a bottom - up fashion, and that this consumes processing resources that could otherwise be used for tracking targets. Störmer et al (2011) found that even distractors in the opposite visual hemifield from where the targets are located interfere with tracking, which suggests that attention may be involuntarily attracted to any moving object, even if it is not confusable with the targets. If the reason that distractors affect tracking is that they attract attention due to their salience, then even when distractors differ in appearance from targets they should still hinder tracking.

Based on the visual-search literature (eg Treisman and Sato 1990; Wolfe et al 1989), it could be posited that in MOT when distractors have different features from targets, the distractors will be filtered out preattentively. Pylyshyn et al (2008) used a probe-dot detection task during tracking, and found that identical distractors were attentionally suppressed, but stationary distractors and distractors in a different depth plane than targets were not attentionally suppressed. Pylyshyn et al proposed that distractors differing from targets on particular features may be filtered out preattentively, and thus active attentional suppression of these distractors is not necessary. If it is correct that when distractors have different features from targets they do not need to be attentionally suppressed, then they should impair tracking less, because more attentional 
resources will be available for tracking the targets. The present study will measure the effects of these feature differences on tracking performance to test this prediction.

A final issue of interest is the nature of the top-down settings for target features during MOT. One hypothesis is that the top-down processes in tracking have independent settings for each individual feature of the targets (color, shape, motion, etc). Some models of visual search have proposed that each feature is represented in a separate feature map, with top-down activation at each location of the map based on how similar the features at that location are to the target features (eg Cave 1999; Wolfe 1994). The top-down influence on attentional allocation is then determined by summing the activations of the feature maps. One source of empirical support for this idea is experiments that have shown that distractors that differ from the target in two features are rejected more efficiently than distractors that differ from the target in only one feature (eg Quinlan and Humphreys 1987; Wolfe et al 1989). In MOT, if features are treated in this fashion, then it is predicted that the greater the number of features that a distractor shares with a target, the greater the attention the distractor will be allocated. Thus, as the number of feature differences between the distractors and targets is increased, the distractors will produce less interference with tracking. A second hypothesis is that the top-down processes in MOT are set to attend to the conjunction of all features of the targets. If this is the case, then attention will only be allocated to distractors that have all of the features of the targets. Distractors that are different from targets on at least one feature will not be allocated attention, but the number of features on which the distractors and targets differ will not matter.

Much attention research has shown that the degree to which distractors are processed depends on the extent to which target processing leaves spare resource capacity (eg Lavie 1995; see Lavie 2005 for a review). When perceptual load is high, almost all perceptual resources must be used for processing targets, so distractors are processed to a limited extent or not at all. However, when perceptual load is low, there are enough resources left over to process distractors to a greater extent. It has been proposed that binding of features into an integrated object requires attentional resources (eg Treisman and Gelade 1980). Evidence for this proposition has come from studies showing that deficits in binding occur when attention is diverted by a demanding task (eg Brown and Brockmole 2010), although some other studies have found the contrary (eg Allen et al 2006). Fougnie and Marois (2009) found that performing an attention-demanding MOT task reduces memory for bound features to a greater extent than memory for individual features. These previous results suggest that perceptual load might affect the ability to use information about multiple bound features of a distractor in MOT.

In view of the previous literature, it is not yet known whether the extent to which distractors hinder MOT is dependent on the features of the distractors. Answering this question is important for two reasons. First, it has significant consequences for theories of MOT. Recently several theories of MOT have proposed that a major reason, perhaps even the primary reason, that distractors interfere with tracking is due to being mistaken for targets (eg Alvarez and Franconeri 2007; Franconeri et al 2010; Intriligator and Cavanagh 2001; Oksama and Hyönä 2004; Pylyshyn 2004). A crucial test of this hypothesis is whether distractors that are less easily confusable with targets will interfere less with tracking. Second, it will allow us to ascertain whether top-down settings for target features have similar effects on MOT mechanisms as they do on visual search mechanisms. Although many studies have shown that during visual search the effect of a distractor object is dependent on whether the distractor shares features with the target (eg Folk et al 1992; Treisman and Sato 1990; Wolfe et al 1989), it is unknown whether this result generalizes to MOT. Consequently, the goal of the present study is to investigate whether distractors' effects on tracking are dependent on the similarity of distractors to targets. 
In experiment 1 , observers tracked 5 targets moving among 5 distractors that were identical to the targets and a number of additional distractors $(0,1,2,5$, or 10$)$ that could have similar or different characteristics from the targets. Experiment 1 investigated whether distractors that have a different feature (color, shape, or motion) from targets impair tracking less than do distractors that are identical to targets. Experiment 2 examined whether distractors that are distinct from targets on two features impair tracking less than distractors that are distinct from targets on only one feature. The target tracking load was also varied in experiment 2 .

\section{Experiment 1}

The purpose of experiment 1 was to test whether distractors hinder tracking less if they have a distinct feature from the targets. If the interfering effect of distractors on tracking ability is contingent on the similarity of distractor features to target features, then it will also be of interest to examine what types of feature information can be used to distinguish distractors from targets. Since previous research has suggested that color information is acquired for tracked objects during MOT (Bahrami 2003; Makovski and Jiang 2009b), it is expected that the visual system should be able to preattentively filter out distractors that differ in color from targets, improving tracking performance. Previous research has also suggested that motion information about targets can be used during MOT (eg Yantis 1992), and that stationary distractors are preattentively filtered out (Pylyshyn et al 2008). However, previous studies regarding whether shape information is acquired during tracking have shown mixed results, with several studies suggesting that it is not acquired (Makovski and Jiang 2009a, control experiment 3; Pylyshyn et al 2008), and others suggesting that at least some shape information is acquired (Bahrami 2003; Horowitz et al 2007). If shape information is accessed during MOT, then the visual system may be able to preattentively filter out distractors that are a different shape from targets, which would improve tracking performance. Some visual search studies have suggested that shape information can be distinguished at a preattentive level (eg Treisman and Gelade 1980). However, a probedot detection task during MOT found that distractors that had a different shape from targets were attentionally suppressed, suggesting that shape information was not used to preattentively filter out the distractors (Pylyshyn et al 2008). It may be that the MOT studies that have not found an effect of shape have used shapes that were not sufficiently distinct, thus the present experiment utilizes shapes that are very dissimilar (disks and crosses). Experiments 1a, 1b, and 1c examined distractors differing from targets based on color, shape, and motion, respectively (see figures 1 and 2).

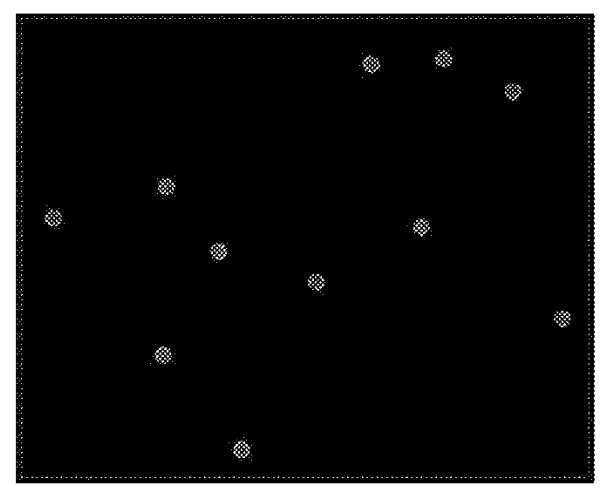

(a)

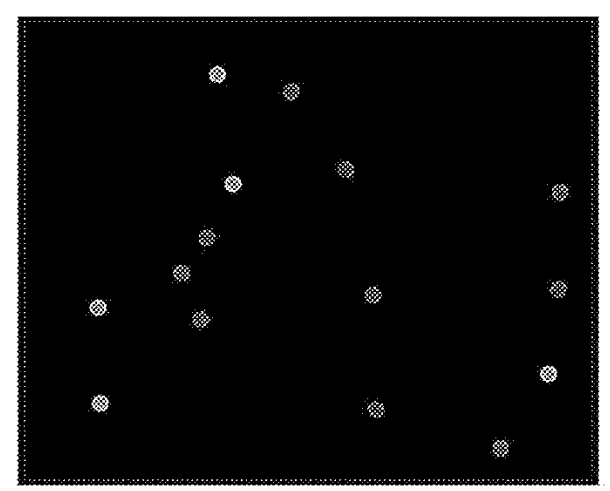

(b)

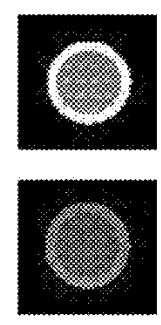

(c)

Figure 1. Examples of the stimuli used in experiment 1a. (a) Display with one same-color additional distractor. (b) Display with five different-color additional distractors. (c) Enlarged views of disks. Different colors in the actual displays are shown here as different shades of gray. 


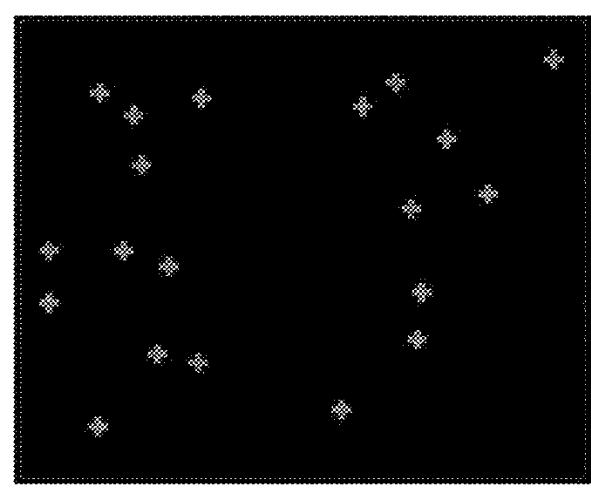

(a)

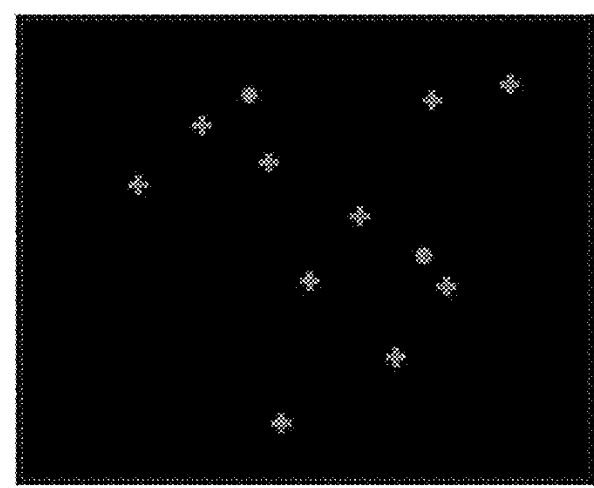

(b)

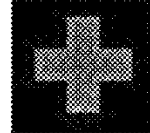

(c)

Figure 2. Examples of the stimuli used in experiment $1 \mathrm{~b}$. (a) Display with ten same-shape additional distractors. (b) Display with two different-shape additional distractors. (c) Enlarged view of a cross.

\section{2a Experiment 1a}

\section{2a.1 Method}

2a.1.1 Observers. Observers were undergraduate students from San Jose State University. Each participated in a $1 \mathrm{~h}$ long session and was compensated with course credit. All observers had self-reported normal color vision and normal or corrected-to-normal visual acuity. None of the observers was familiar with the purpose of the experiment. There were thirty-two observers in experiment la.

2a.1.2 Apparatus. Observers were seated in a darkened room approximately $57 \mathrm{~cm}$ from the display. The stimuli were presented on a $50 \mathrm{~cm}$ flat-screen CRT monitor with a pixel resolution of $1920 \times 1440$, controlled by a Dell Precision workstation. The experimental procedure was generated in $\mathrm{C}++$, using the OpenGL libraries.

2a.1.3 Stimuli. On each trial, several disks were presented on a black background (see figure 1). On every trial, there were 5 targets that were all the same color, and 5 identical distractors that were the same color as the targets. Depending on the trial, there was also a number of additional distractors $(0,1,2,5$, or 10). The additional distractors were either the same color as the targets, or a different color.

At the beginning of each trial, several stationary disks were presented on the monitor at randomly chosen locations, with the constraint that the disks could not overlap. 5 of the disks flashed on and off 5 times over a period of $2.5 \mathrm{~s}$, to designate them as the targets. Next all the disks moved about the screen for $6 \mathrm{~s}$. Each disk had a speed of $3.7 \mathrm{deg} \mathrm{s}^{-1}$ and a different randomly chosen direction. The motion trajectories of the disks were linear. The $x$ - and $y$-components of the speed were chosen randomly for each disk, with the constraint that both the $x$ - and $y$-components had to be at least $0.18 \mathrm{deg} \mathrm{s}^{-1}$. Each of these speed components remained constant throughout the trial. Disks could intersect each other during the motion. After the end of the motion, one disk was probed by changing its color to red, and the observer responded whether it was a target or a distractor by pressing a mouse button. The probed disk was randomly selected only out of the 5 targets and 5 identical distractors, with an equal probability of being a target or a distractor.

Each disk had a diameter of $0.74 \mathrm{deg}$ visual angle, including an outline of width $0.08 \mathrm{deg}$. The two possible colors for the disks were green and blue. Green disks consisted of a darker green inner area and lighter green outline. Blue disks consisted of a darker blue inner area and lighter blue outline. The purpose of the outlines was to provide an occlusion cue ( $\mathrm{T}$-junction information) specifying which disk was in front 
when disks' trajectories overlapped. Previous research has found that when object boundaries intersect, tracking performance is better if T-junctions are present than if no T-junctions are present (Viswanathan and Mingolla 2002). The depth order of the disks was assigned randomly at the beginning of each trial.

The display area was bounded by a gray outline rectangle that subtended $26 \mathrm{deg} \times 21 \mathrm{deg}$. The lines composing the rectangle were $0.04 \mathrm{deg}$ in width ( 2 pixels). There was a buffer around each line of the rectangle, so that the disks would not touch the rectangle. Whenever the center of a disk reached $1.0 \mathrm{deg}$ from the edge of the display area, the disk's direction was reversed to send it back into the display area.

2a.1.4 Procedure. Observers were instructed to track the target disks during the motion, and at the end of the motion to respond whether the red probed disk was a target or a distractor by pressing a mouse button. After responding, observers were given feedback either that their response was correct or incorrect. After viewing the feedback, observers pressed a key to move on to the next trial.

2a.1.5 Design. The independent variables were color of additional distractors (same as targets, different from targets) and number of additional distractors $(0,1,2,5,10)$. Both independent variables were run within-subjects. Target color was counterbalanced across observers - for half of the observers the targets were blue and the different-color additional distractors were green, while for the other half of the observers the targets were green and the different-color additional distractors were blue.

The dependent variable was the proportion of correct responses in identifying whether the red probed disk was a target or a distractor. Each observer completed two blocks. The first block consisted of 18 practice trials followed by 72 experimental trials, and the second block consisted of 5 practice trials followed by 72 experimental trials. Each block contained 8 experimental trials in each of the 9 conditions. Within each block, the trials were presented in a randomly intermixed order for each observer.

\section{2a.2 Results}

The mean proportion of correct responses was calculated for each observer for each of the 9 conditions. The data from the 8 conditions on which additional distractors were present were submitted to a repeated-measures analysis of variance (ANOVA) including the factors of color of additional distractors (same as targets, different from targets) and number of additional distractors $(1,2,5,10)$. The data from the 0 additional-distractor condition were not included in the ANOVA, because it is not possible to have levels of the additional-distractor color variable when no additional distractors are present. Figure $3 \mathrm{a}$ depicts the results.

The main effect of color of additional distractors was significant $\left(F_{1,31}=5.78\right.$, $p=0.022, \eta^{2}=0.16$ ). Tracking performance was higher with different-color additional distractors $(M=0.77, \mathrm{SD}=0.10)$ than with same-color additional distractors $(M=0.73, \mathrm{SD}=0.11)$. The main effect of number of additional distractors was significant $\left(F_{2.8,85.6}=8.07, p<0.001, \eta^{2}=0.21\right)$, in that tracking performance declined as number of additional distractors increased. (Note: All tests of within-subjects independent variables with more than two levels were adjusted using the Greenhouse-Geisser procedure, to compensate for possible deviations from sphericity.) There was no significant interaction between color and number of additional distractors $\left(F_{2.8,86.4}=0.39\right.$, $p=0.743$ ). Since the 0 additional-distractor condition was not part of the ANOVA, $t$-tests were performed comparing the 0 additional-distractor condition with each of the other 8 conditions, using an $\alpha$ level of 0.05 . Significant differences were found between the 0 additional distractor condition and the 5 and 10 different-color and 5 and 10 same-color additional-distractor conditions. 

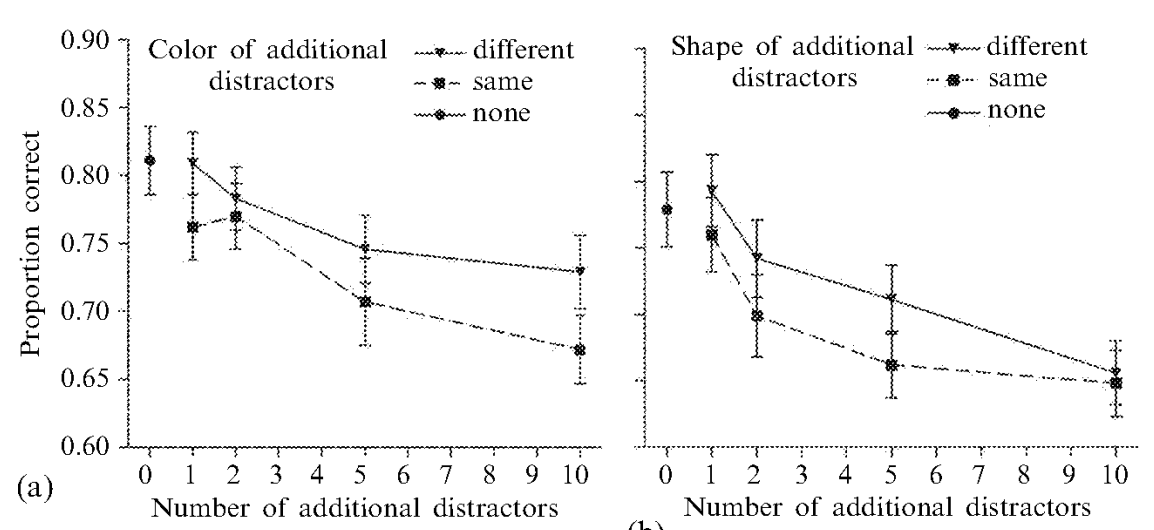

(b)

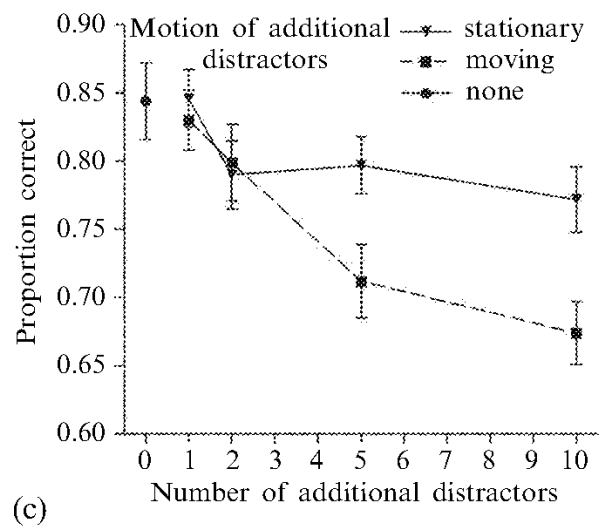

Figure 3. Experiment 1 data. (a) Proportion correct as a function of color of additional distractors and number of additional distractors in experiment la. Error bars represent standard error. (b) Proportion correct as a function of shape of additional distractors and number of additional distractors in experiment $1 \mathrm{~b}$. (c) Proportion correct as a function of motion of additional distractors and number of additional distractors in experiment $1 \mathrm{c}$.

A possible alternative explanation for the reduction in proportion correct tracking performance with increasing number of additional distractors could be that the observer's response criterion was biased by the number of distractors. That is, at the end of each trial when one disk was probed and the observer had to respond whether that disk was a target or a distractor, although the probed disk was equally likely to be a target or a distractor, the observer might have been biased to respond more conservatively (ie more likely to respond "distractor") when more distractors were present. In order to test this explanation, sensitivity $\left(d^{\prime}\right)$ and response criterion $(\beta)$ measures from signal-detection theory were calculated (eg Stanislaw and Todorov 1999). Trials on which the observer correctly indicated that the probed disk was a target were coded as hits, and trials on which a distractor disk was probed but the observer incorrectly indicated that it was a target were coded as false alarms. On the basis of these measurements, $d^{\prime}$ and $\beta$ were computed for each observer for each of the conditions, and ANOVAs were conducted. The analysis of $\beta$ showed no significant main effect of number of additional distractors $\left(F_{2.8,86.8}=1.50, p=0.222\right)$, no significant main effect of color of additional distractors $\left(F_{1,31}=0.43, p=0.516\right)$, and no significant interaction between color and number of additional distractors $\left(F_{2.8,85.4}=1.45\right.$, $p=0.236$ ). On the other hand, the analysis of $d^{\prime}$ showed a significant main effect of number of additional distractors $\left(F_{2.7,84.8}=6.47, p=0.001, \eta^{2}=0.17\right)$ and a significant main effect of color of additional distractors $\left(F_{1,31}=5.59, p=0.024, \eta^{2}=0.15\right)$, but no significant interaction between color and number of additional distractors $\left(F_{2.7,82.3}=0.42, p=0.718\right)$. These $d^{\prime}$ results follow the same patterns as the results found in the proportion correct data. The finding that $\beta$ is not affected by number and color of additional distractors, and that $d^{\prime}$ is affected by these manipulations, suggests that the effects of these manipulations on proportion correct are not due to biasing the response criterion. 


\section{2b Experiment 1b}

2b.1 Method

The method was the same as in experiment 1a, except as noted below.

2b.1.1 Observers. There were thirty-two observers in experiment $1 b$.

2b.1.2 Stimuli. Both the target and distractor objects could be disks or crosses, and all objects were green in color (see figure 2). On every trial, there were 5 targets that were all the same shape, and 5 identical distractors that were the same shape as the targets. Depending on the trial, there was also a number of additional distractors, which were either the same shape as the targets, or a different shape. Each cross had a height and length of $0.86 \mathrm{deg}$, including an outline of width $0.06 \mathrm{deg}$. The width of each bar of the cross was $0.29 \mathrm{deg}$. These dimensions made the total area of a cross equivalent to the total area of a disk. On each cross, the inner area was darker green and the outline was lighter green.

2b.1.3 Design. The independent variables were shape of additional distractors (same as targets, different from targets) and number of additional distractors $(0,1,2,5,10)$. Target shape was counterbalanced across observers - for half of the observers the targets were disks and the different-shape additional distractors were crosses, while for the other half of the observers the targets were crosses and the different-shape additional distractors were disks.

\section{2b.2 Results}

Figure $3 \mathrm{~b}$ depicts the results. The main effect of shape of additional distractors was significant $\left(F_{1,31}=4.48, p=0.042, \eta^{2}=0.13\right)$. Tracking performance was higher with different-shape additional distractors $(M=0.73, \mathrm{SD}=0.12)$ than with same-shape additional distractors $(M=0.69, \mathrm{SD}=0.12)$. The main effect of number of additional distractors was significant $\left(F_{2.8,86.4}=13.57, p<0.001, \eta^{2}=0.30\right)$. There was no significant interaction between shape and number of additional distractors $\left(F_{2.6,81.4}=0.62\right.$, $p=0.586$ ). The 0 additional-distractor condition was compared with each of the other 8 conditions using $t$-tests. Significant differences were found between the 0 additionaldistractor condition and the 5 and 10 different-shape and 2, 5, and 10 same-shape additional-distractor conditions.

\section{2c Experiment 1c}

\section{2c.1 Method}

The method was the same as in experiment 1a, except as noted below.

2c.1.1 Observers. There were twenty-eight observers in experiment 1c.

2c.1.2 Stimuli. Each observer was presented with all disks in a single color. On every trial, there were 5 moving targets, and 5 moving identical distractors. Depending on the trial, there was also a number of additional distractors, which were either moving or stationary. During the $6 \mathrm{~s}$ motion period, the moving disks moved as in experiment 1a, but the stationary disks did not move at all. Because the depth order of the disks was assigned randomly at the beginning of each trial, when the trajectory of a moving disk intersected a stationary disk, the moving disk could appear to be either in front of or behind the stationary disk.

2c.1.3 Design. The independent variables were motion of additional distractors (moving, stationary) and number of additional distractors $(0,1,2,5,10)$. For half of the observers all the disks were blue, while for the other half of the observers all the disks were green. 


\section{2c.2 Results}

Figure $3 \mathrm{c}$ depicts the results. The main effect of motion of additional distractors was significant $\left(F_{1,27}=10.84, p=0.003, \eta^{2}=0.29\right)$. Tracking performance was higher with stationary additional distractors $(M=0.80, \mathrm{SD}=0.08)$ than with moving additional distractors $(M=0.75, \mathrm{SD}=0.10)$. The main effect of number of additional distractors was significant $\left(F_{2.8,76.9}=13.57, p<0.001, \eta^{2}=0.34\right)$.

The interaction between motion and number of additional distractors was also significant $\left(F_{2.5,68.5}=3.67, p=0.022, \eta^{2}=0.12\right)$. Fisher least significant difference (LSD) comparisons indicated that when there were 1 or 2 additional distractors, tracking performance did not differ significantly between moving and stationary additional distractors $(p \mathrm{~s}>0.05)$. However, when there were 5 or 10 additional distractors, tracking performance was higher with stationary than with moving additional distractors $(p s<0.01)$. A separate ANOVA was performed on only the stationary additionaldistractor data. For stationary additional distractors, the main effect of number of additional distractors approached significance $\left(F_{2.7,73.1}=2.80, p=0.051, \eta^{2}=0.09\right)$. The 0 additional-distractor condition was compared with each of the other 8 conditions using $t$-tests. Significant differences were found between the 0 additional-distractor condition and the 10 stationary and 5 and 10 moving additional-distractor conditions.

An additional ANOVA was also conducted to compare the interaction found in experiment $1 \mathrm{c}$ with the null interactions of experiments $1 \mathrm{a}$ and $1 \mathrm{~b}$, with experiment (ie distractor feature) as a between-subjects factor. However, the three-way interaction between experiment, number of additional distractors, and same/different additional distractors was not significant $\left(F_{60,265.7}=1.53, p=0.169\right)$.

\section{2c.3 Discussion}

In experiment 1, tracking performance was higher when the additional distractors had a distinct feature from the targets than when they had the same features as the targets. This demonstrates that in MOT, distractor objects that do not share the features of the targets interfere less with tracking, indicating that top-down processing plays a role in the mechanisms by which distractors hinder tracking. This result is indicative of top-down processing because, based on the observer's knowledge that targets have a particular feature, items that do not have that feature can be identified as being distractors. The present findings are consistent with many studies on attention capture and visual search that have found that distractor objects that do not share the features of the targets do not interfere with search (eg Folk et al 1992; Folk and Remington 1998; Treisman and Sato 1990; Wolfe et al 1989).

One explanation for why different-feature distractors interfere less with tracking than same-feature distractors is that when a different-feature distractor passes close to a target, it is less likely to be mistaken for the target. However, this may not be the only reason for the distractor similarity effect. Störmer et al (2011) found that while tracking targets within one visual hemifield, the presence of distractors in the opposite hemifield interfered with tracking, but this interference was reduced when the distractors were a different color from the targets. The fact that distractor feature similarity affects target tracking even for distractors that never come near targets, and thus are unlikely to be confused with targets, lends support to the idea of feature-dependent attentional suppression of distractors that occurs regardless of location.

Interestingly, in experiments $1 \mathrm{a}$ and $1 \mathrm{~b}$ tracking performance declined as the number of additional distractors increased, both for same-feature and different-feature additional distractors. The result that tracking declined as the number of same-feature additional distractors increased is consistent with previous studies (Bettencourt and Somers 2009; Sears and Pylyshyn 2000). More importantly, the decline in tracking as the number of different-feature additional distractors increased indicates that even 
distractors that have a distinct feature from the targets produce some interference with tracking. Thus, it does not appear that distractors that have a different feature from targets are completely filtered out at a preattentive level. One possible explanation for this is that preattentive filtering is graded, and distinct distractors are filtered to some incomplete extent. Another possibility is that only a subset of the distractors were preattentively filtered out. Preattentive filtering of a distractor should only be possible if the distractor's features are acquired, and some previous studies have suggested that feature information is acquired for only a subset of the objects during tracking (Botterill et al 2011; Cohen et al 2011; Fencsik et al 2007; Horowitz et al 2007; Ren et al 2009). The finding that even distinct distractors produce some interference with tracking is also consistent with a role for bottom - up processes in the interfering effect of distractors in MOT, in which distractors divert attention away from targets due to their physical salience (Bettencourt and Somers 2009).

In experiment $1 \mathrm{c}$, however, there was a significant interaction, indicating that tracking performance was affected less by the number of additional distractors when the additional distractors were stationary than when they were moving. This result supports Pylyshyn et al's (2008) assertion that stationary distractors are, to a large extent, preattentively filtered out during MOT. This result is also consistent with Pinto et al's (2006) proposition that attention to moving and stationary objects is based on separate transient and sustained processing channels.

Notably, the presence of a single different-color, different-shape, or stationary additional distractor did not impair tracking, relative to no additional distractors. Given the literature on attention capture, it might have been expected that a single differentfeature distractor would have been highly salient and attracted attention. During visual search, the presence of a different-feature singleton often attracts attention involuntarily (eg Theeuwes 1992, 1994). Some theories of visual search characterize bottom - up activation as how different an object is from surrounding objects (eg Cave 1999; Wolfe 1994), so a single unique object would be expected to be highly salient. The finding that a different-feature singleton did not interfere with tracking suggests that what constitutes a 'salient' object may be different in MOT than in visual search, presumably due to the more temporally sustained nature of the task and the attentional settings for motion. It is important to note, however, that attention capture by a different-color or different-shape distractor might have occurred during the target designation period before the beginning of the motion, whereas attention capture by a stationary distractor would occur after objects began to move. Perhaps in experiment $1 \mathrm{c}$ a single stationary distractor impaired tracking as much as a single moving distractor owing to the single stationary distractor capturing attention.

It could be contended that tracking performance might be improved when additional distractors have a different feature from the targets simply due to tracking being facilitated when objects are more heterogeneous. However, we believe this explanation to be unlikely. Makovski and Jiang (2009b) found that tracking performance was not better when half of the targets and half of the distractors were one color and the other objects another color than when all objects were identical, suggesting that tracking is not improved merely by the presence of multiple colors.

\section{Experiment 2}

Experiment 1 found that top-down processing plays a role in the effect of distractors on MOT and that the features of color, shape, and motion can be used to distinguish distractors from targets. Yet it also indicates that even distinct distractors produce some interference with tracking. These results suggest that distractors that have a different feature from targets may be partially, rather than completely, filtered out at a preattentive level. However, the distractors differed from targets only on a single feature. 
Perhaps if distractors are more distinct from targets, they could be completely filtered out preattentively, and have no effect whatsoever on tracking. Experiment 2 was carried out to test whether distractors that are distinct from targets on two features interfere even less with tracking than distractors that are distinct from targets on a single feature.

One hypothesis is that the top-down processes in MOT have independent settings for each individual feature of the targets, similar to the additive feature maps posited for visual search (eg Cave 1999; Wolfe 1994). If this is the case, then tracking performance will be higher with additional distractors that differ from targets on two features than with additional distractors that differ on only one feature. A second hypothesis is that the top-down processes in tracking are set to attend to the conjunction of the targets' features, and thus only distractors that have all of the targets' features will receive attention. If this is the case, then tracking performance will be equal for additional distractors that differ from targets on one feature and on two features.

Two versions of experiment 2 were conducted, one examining the conjunction of shape and color, and the other examining the conjunction of motion and color. In experiments $2 \mathrm{a}, 2 \mathrm{c}$, and $2 \mathrm{~d}$, the additional distractors were a different shape from the targets on every trial, and could be either the same color as the targets or a different color from the targets. In experiment $2 b$, the additional distractors were stationary on every trial, and could be either the same color as the targets or a different color from the targets.

Because the amount of attention available for processing distractors may be affected by the amount of resources used for processing targets (eg Lavie 1995), and the process of feature binding requires attentional resources (eg Brown and Brockmole 2010; Fougnie and Marois 2009; Treisman and Gelade 1980), the ability to use information about multiple features of distractors in MOT might be constrained by target tracking load. Thus, target tracking load was manipulated in experiment 2 . The number of targets to be tracked was 5 in experiments $2 \mathrm{a}$ and $2 \mathrm{~b}, 3$ in experiment $2 \mathrm{c}$, and 2 in experiment $2 \mathrm{~d}$. Motion speed was increased as number of targets was reduced, in order to avoid ceiling effects.

\section{3a Experiment 2a}

3a.1 Method

The method was the same as in experiment 1a, except as noted below.

3a.1.1 Observers. There were forty-four observers in experiment $2 \mathrm{a}$.

3a.1.2 Stimuli. On every trial, there were 5 targets that were all the same color and shape, and 5 identical distractors that were the same color and shape as the targets. Depending on the trial, there was also a number of additional distractors, which were either the same color as the targets, or a different color. The additional distractors were a different shape from the targets on every trial.

3a.1.3 Design. Target color and target shape were both counterbalanced across observers. One-quarter of the observers was in each of the following conditions: (a) targets were blue disks and the additional distractors were blue or green crosses, (b) targets were blue crosses and the additional distractors were blue or green disks, (c) targets were green disks and the additional distractors were green or blue crosses, and (d) targets were green crosses and the additional distractors were green or blue disks.

\section{3a.2 Results}

Figure 4a depicts the results. There was no significant main effect of color of additional distractors $\left(F_{1,43}=2.03, p=0.161\right)$. Tracking performance did not differ for different-color additional distractors $(M=0.79, \mathrm{SD}=0.11)$ and same-color additional distractors $(M=0.77, \mathrm{SD}=0.11)$. The main effect of number of additional distractors 

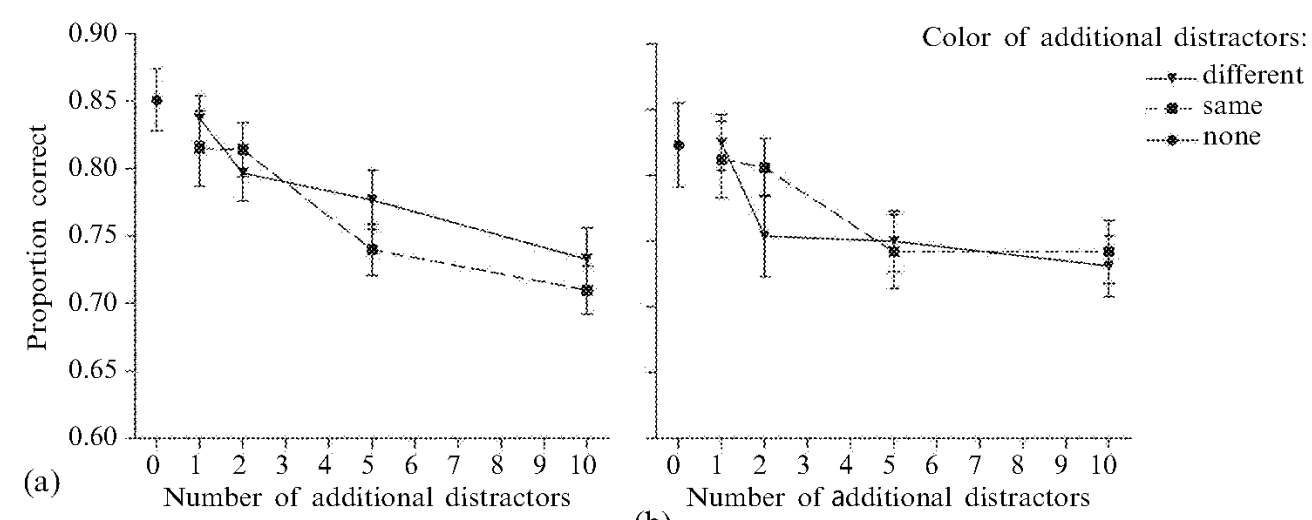

(b)
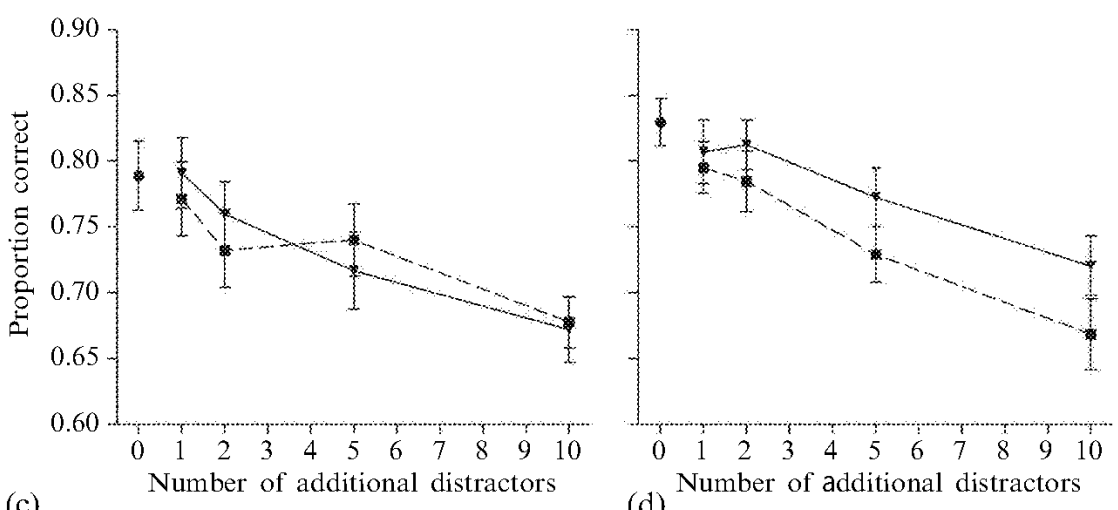

Figure 4. Experiment 2 data. Proportion correct as a function of color of additional distractors and number of additional distractors in (a) experiment $2 \mathrm{a}$, in which the additional distractors were a different shape from the targets on every trial and there were 5 targets; (b) experiment $2 \mathrm{~b}$, in which the additional distractors were stationary on every trial and there were 5 targets; (c) experiment $2 \mathrm{c}$, in which the additional distractors were a different shape from the targets on every trial and there were 3 targets; and (d) experiment $2 \mathrm{~d}$, in which the additional distractors were a different shape from the targets on every trial and there were 2 targets.

was significant $\left(F_{2.7,117.9}=16.49, p<0.001, \eta^{2}=0.28\right)$. There was no significant interaction between color and number of additional distractors $\left(F_{2.8,119.1}=1.25, p=0.295\right)$. The 0 additional-distractor condition was compared with each of the other 8 conditions using $t$-tests. Significant differences were found between the 0 additional-distractor condition and the 2, 5, and 10 different-color and 5 and 10 same-color additionaldistractor conditions.

\section{3b Experiment 2b}

3b.1 Method

The method was the same as in experiment 1a, except as noted below.

\section{3b.1.1 Observers. There were thirty observers in experiment $2 b$.}

3b.1.2 Stimuli. The stimuli were the same as in experiment 1c, with the following exceptions. On every trial, there were 5 targets that were all the same color, and 5 identical distractors that were the same color as the targets. The targets and identical distractors were moving on every trial. Depending on the trial, there was also a number of additional distractors, which were either the same color as the targets, or a different color. The additional distractors were stationary on every trial. 


\section{3b.2 Results}

Figure $4 \mathrm{~b}$ depicts the results. There was no significant main effect of color of additional distractors $\left(F_{1,29}=0.57, p=0.456\right)$. Tracking performance did not differ for differentcolor additional distractors $(M=0.77, \mathrm{SD}=0.10)$ and same-color additional distractors $(M=0.78, \mathrm{SD}=0.10)$. The main effect of number of additional distractors was significant $\left(F_{2.88,80.2}=8.57, p<0.001, \eta^{2}=0.23\right)$. There was no significant interaction between color and number of additional distractors $\left(F_{2.5,72.3}=1.01, p=0.382\right)$. The 0 additionaldistractor condition was compared with each of the other 8 conditions using $t$-tests. Significant differences were found between the 0 additional-distractor condition and the 2, 5, and 10 different-color and 5 and 10 same-color additional-distractor conditions.

\section{3c Experiment 2c}

\section{3c.1 Method}

The method was the same as in experiment $2 \mathrm{a}$, except as noted below.

3c.1.1 Observers. There were thirty-two observers in experiment $2 \mathrm{c}$.

3c.1.2 Stimuli. On every trial, there were 3 targets that were all the same color and shape, and 5 identical distractors that were the same color and shape as the targets. During the motion period, each disk had a speed of $11.1 \mathrm{deg} \mathrm{s}^{-1}$, and both the $x$ - and $y$-components of the speed had to be at least $0.55 \mathrm{deg} \mathrm{s}^{-1}$.

\section{3c.2 Results}

Figure $4 \mathrm{c}$ depicts the results. There was no significant main effect of color of additional distractors $\left(F_{1,31}=0.11, p=0.747\right)$. Tracking performance did not differ for differentcolor additional distractors $(M=0.74, \mathrm{SD}=0.12)$ and same-color additional distractors $(M=0.73, \mathrm{SD}=0.11)$. The main effect of number of additional distractors was significant $\left(F_{2.8,85.8}=8.72, p<0.001, \eta^{2}=0.22\right)$. There was no significant interaction between color and number of additional distractors $\left(F_{2.7,84.5}=0.81, p=0.484\right)$. The 0 additionaldistractor condition was compared with each of the other 8 conditions using $t$-tests. Significant differences were found between the 0 additional-distractor condition and the 5 and 10 different-color and 10 same-color additional-distractor conditions.

\section{3d Experiment 2d}

3d.1 Method

The method was the same as in experiment $2 \mathrm{a}$, except as noted below.

3d.1.1 Observers. There were thirty-six observers in experiment $2 \mathrm{~d}$.

3d.1.2 Stimuli. On every trial, there were 2 targets that were all the same color and shape, and 5 identical distractors that were the same color and shape as the targets. During the motion period, each disk had a speed of $18.5 \mathrm{deg} \mathrm{s}^{-1}$, and both the $x$ - and $y$-components of the speed had to be at least $0.92 \mathrm{deg} \mathrm{s}^{-1}$.

\section{$3 \mathrm{~d} .2$ Results}

Figure $4 \mathrm{~d}$ depicts the results. The main effect of color of additional distractors was significant $\left(F_{1,35}=5.77, p=0.022, \eta^{2}=0.14\right)$. Tracking performance was higher with different-color additional distractors $(M=0.78, \mathrm{SD}=0.10)$ than with same-color additional distractors $(M=0.74, \mathrm{SD}=0.10)$. The main effect of number of additional distractors was significant $\left(F_{2.5,86.5}=16.22, p<0.001, \eta^{2}=0.32\right)$. There was no significant interaction between color and number of additional distractors $\left(F_{2.9,100.6}=0.55\right.$, $p=0.641$ ). The 0 additional-distractor condition was compared with each of the other 8 conditions using $t$-tests. Significant differences were found between the 0 additional-distractor condition and the 5 and 10 different-color and 5 and 10 same-color additional-distractor conditions. 


\subsection{Discussion}

In experiments $2 \mathrm{a}$ and $2 \mathrm{c}$, with 5 targets or 3 targets to be tracked, tracking performance was not higher with different-color, different-shape additional distractors than with same-color, different-shape additional distractors. Similarly, in experiment $2 \mathrm{~b}$, with 5 targets, tracking performance was not higher with different-color stationary additional distractors than with same-color stationary additional distractors. However, when the number of targets was reduced to 2 , in experiment $2 \mathrm{~d}$, tracking performance was higher with additional distractors that differed from targets on two features than with those that differed on one feature. These findings demonstrate that distractors that differ from targets on two features hinder tracking less than distractors that differ from targets on a single feature, but only when target tracking load is low. A low tracking load draws less on attentional resources, presumably leaving more resources available for processing multiple distractor features. This result suggests that during MOT each individual feature of the targets is represented by independent settings, similar to the additive feature maps posited for visual search (eg Cave 1999; Wolfe 1994), but that it may not be possible to use several of these feature maps simultaneously unless adequate processing resources are available.

In experiment 2, regardless of the number of targets to be tracked, tracking performance decreased as the number of additional distractors increased, even for different-color, different-shape additional distractors and for different-color stationary additional distractors. This shows that even distractors that are distinct from targets on two features hinder tracking, suggesting that they are not completely filtered out at a preattentive level. This result is also consistent with a role for salience in the interfering effect of distractors in tracking (Bettencourt and Somers 2009).

\section{General discussion}

The goal of the present study was to examine whether the extent to which distractors impair MOT is affected by the feature similarity of the distractors to the targets. Experiment 1 established the principal finding, that distractors that have a distinct feature (shape, color, or motion) from the targets hinder tracking less than distractors that are identical to the targets. This result suggests that distractors' interfering effects on tracking are dependent on top-down settings for target features. Previous studies on visual search and attention capture have found that the effect of a distractor object is dependent on the distractor object sharing the features of the target (eg Folk et al 1992; Folk and Remington 1998; Treisman and Sato 1990; Wolfe et al 1989), and the current study demonstrates that this result generalizes to MOT.

However, experiment 1 also showed that tracking performance declined as the number of additional distractors increased, even when the additional distractors were different in color or shape from the targets. Experiment 2 showed that even when distractors were distinct from targets on two features (shape and color in experiments $2 \mathrm{a}, 2 \mathrm{c}$, and $2 \mathrm{~d}$, or motion and color in experiment $2 \mathrm{~b}$ ), tracking performance still decreased as the number of additional distractors increased. These results indicate that even featurally distinct distractors still interfere with tracking, albeit to a reduced extent. Thus, it does not appear that distractors with distinct features from targets are completely filtered out preattentively.

Two main mechanisms that have been proposed for how distractors might interfere with tracking are (a) that when a distractor passes near a target, the observer sometimes mistakes the distractor for the target (Alvarez and Franconeri 2007; Bettencourt and Somers 2009; Horowitz et al 2007; Iordanescu et al 2009; Intriligator and Cavanagh 2001; Oksama and Hyönä 2004; Pylyshyn 2004; Sears and Pylyshyn 2000), and (b) that distractors may divert attention away from tracking the targets due to their physical salience (Bettencourt and Somers 2009). The present results suggest that both of these 
mechanisms contribute to the interfering effects of distractors. The finding that reducing the similarity of distractors to targets reduces their interference is consistent with the idea that distractors affect tracking because they are confused with targets. This result also suggests that the process of individuating targets and distractors that are nearby each other takes into account top-down knowledge of target features. On the other hand, the finding that even distinct distractors produce some interference with tracking suggests that the presence of any physically salient object may attract attention away from the targets.

There are at least two explanations for how a feature difference might reduce confusions between distractors and targets. Previous studies have found that the attentional focus on each target in MOT has a limited spatial resolution, and when the distance between a target and a distractor is smaller than the spatial resolution, it becomes difficult to select only the target (Intriligator and Cavanagh 2001). The presence of a feature difference might improve tracking by allowing the observer to tolerate a closer distance between a target and distractor. Another possibility is that feature-difference information is used in attempting to recover lost targets (see Horowitz et al 2007; Makovski and Jiang 2009b). That is, when a target is lost, knowledge of targets' features could help to distinguish which objects could most likely have been the lost target. Future studies are needed to examine to what extent each of these mechanisms contributes to the feature-based effects.

Experiment 2 showed that distractors that are distinct from targets on two features interfere less with tracking than distractors that are distinct from targets on a single feature, when tracking load is low. This finding is inconsistent with the hypothesis that the top-down processes in tracking are set to attend to the conjunction of the targets' features and that only distractors that have all of the targets' features will receive attention, because the number of features on which the distractors differed from targets affected the attentional allocation. This result is instead comparable to findings that during visual search, distractors that differ from the target on two features are rejected more efficiently than distractors that differ on only one feature (Quinlan and Humphreys 1987; Wolfe et al 1989). It has been proposed that during visual search, attentional allocation is determined by the sum of the activations of feature maps (eg Cave 1999; Wolfe 1994). The present results suggest that top-down attentional allocation in tracking, similarly to visual search, is determined by independent settings for each individual feature of the targets.

The finding that several features of a distractor can simultaneously be used to ignore the distractor is at variance, however, with previous studies that have suggested that when multiple objects are moving, the features of each object are not properly bound (Makovski and Jiang 2009a; Saiki 2003; Saiki and Miyatsuji 2007) as they are with stationary objects (eg Luck and Vogel 1997). If the shape and color of an object are not integrated, then it should be impossible to use information about both features of the object together for top-down processing. One reason for the difference in results may be that whereas the previous studies presented four (Saiki 2003; Saiki and Miyatsuji 2007) or eight objects simultaneously (Makovski and Jiang 2009a), each with a unique feature conjunction, the current study only presented two feature conjunctions simultaneously, each occurring on several objects. This smaller number of feature conjunctions may have required fewer resources for binding, facilitating the attainment of binding. Thus, the present results suggest that feature binding on multiple moving objects is possible, at least under some circumstances.

Interestingly though, experiment 2 showed that with high tracking loads, distractors that are distinct from targets on two features do not interfere less with tracking than distractors that are distinct from targets on a single feature. This suggests that perceptual load affects the ability to use information about multiple bound features of a distractor 
in MOT. This is consistent with previous findings that the amount of resources used for processing targets affects the amount of attention available for processing distractors (eg Lavie 1995), and that feature binding requires attentional resources (eg Brown and Brockmole 2010; Fougnie and Marois 2009; Treisman and Gelade 1980). Thus, during MOT, it may not be possible to use information from multiple feature maps simultaneously unless sufficient resources are available for processing and binding the features. Visual short-term-memory (VSTM) limitations may also be a factor in the lack of a benefit of a second-feature difference. Makovski and Jiang (2009b) found that performing a concurrent color-memory task interfered with tracking and eliminated the advantage of tracking uniquely colored objects over tracking homogeneous objects. This suggested that VSTM shares resources with tracking (see also Fougnie and Marois 2006), and is used to maintain feature information during tracking. VSTM limitations could explain the lack of a benefit of a second-feature distinction, because when the demands of tracking a large number of targets has drained resources away from VSTM, there will not be sufficient VSTM resources available to maintain two features of each distractor.

The findings of the present experiments imply that the visual system has access to featural information about targets and distractors during tracking. Previous studies had been mixed on this issue, with some studies suggesting that feature and identity information can be used during tracking (Makovski and Jiang 2009b; Pylyshyn et al 2008; Yantis 1992), and other studies suggesting that processing of feature and identity information is more limited or occurs for only a subset of the tracked targets (Botterill et al 2011; Cohen et al 2011; Pylyshyn 2004; Ren et al 2009). One notable methodological distinction is that, in several experiments that suggested limited feature acquisition, observers were required to use feature information explicitly, by making a response regarding the features or identity of the targets, rather than just tracking the targets. It may be that observers have limited explicit access to feature information about objects during MOT, so they have difficulty consciously making responses about the features of the objects, although feature information is being accessed at an implicit level (see Horowitz et al 2007). Since the present study indicates that feature information can be used to distinguish distractors from targets, it will be interesting for future research to investigate the extent to which the top-down processes in distractor interference are based on explicit access to the features of each individual distractor.

Another topic of interest is which particular types of feature information are processed during MOT. The present experiments demonstrated that shape, color, and motion information can be used to distinguish distractors from targets. These results are consistent with previous research showing that color information (Makovski and Jiang 2009b) and motion information (Pylyshyn et al 2008; Yantis 1992) are processed for tracked objects during MOT. However, previous studies had found inconsistent results regarding the use of shape information during MOT, with some studies suggesting that shape information is not processed (Makovski and Jiang 2009a; Pylyshyn et al 2008) and others suggesting that it is processed (Horowitz et al 2007). An explanation for these varied results is that shape may be encoded only very coarsely during MOT. Thus gross distinctions between shapes, such as those in the present study, are distinguishable during tracking, but finer distinctions between objects, like complex shapes or relatively similar shapes, may not be distinguishable during tracking.

In summary, the present study demonstrates that the interfering effect of distractors on MOT is dependent on top-down settings for target features. Shape, color, and motion information about distractors can be used to distinguish them from targets during tracking. The study shows that feature binding on multiple moving objects is possible, at least under some circumstances, when perceptual load is low and sufficient attentional resources are available. The results suggest that distractors interfere with tracking both due to being confused with targets, and due to their physical salience. 
Acknowledgments. This research was supported by a California State University Research Funds grant, and grants from the San Jose State University College of Social Sciences, Department of Psychology, and University Planning Council. Part of this research was presented at the annual meeting of the Vision Sciences Society, Naples, Florida, May 2010. I thank Julieta Barragan, Jeffrey Cibotti, Leonna Davis-Ross, Sylvia Gonzalez, Veronica Gunawan, Jocelyn Gutierrez, Jia Liang, Sin Lee Loh, Anne-Marie McReynolds, Shahin Najafi, Martha Ruiz, Brenda Serna, Justin Shaw, Chad Smith, Trevor Southard, Blanca Tapia, Tina Tram, and Jennifer Umayam for assistance with data collection and helpful conversations. For computer programming help, I thank Harpreet Bola. I thank Gilbert Remillard for valuable advice and discussions. I also thank Todd Horowitz for his valuable suggestion, which motivated experiment $2 \mathrm{a}$.

These experiments were carried out in accordance with the relevant institutional and national regulations and legislation for human subjects research and in accordance with the World Medical Association Helsinki Declaration as revised in October 2008.

\section{References}

Allen R J, Baddeley A D, Hitch G J, 2006 "Is the binding of visual features in working memory resource-demanding?" Journal of Experimental Psychology: General $135298-313$

Alvarez G A, Franconeri S L, 2007 "How many objects can you track? Evidence for a resourcelimited attentive tracking mechanism" Journal of Vision 7(13) $1-10$

Bahrami B, 2003 "Object property encoding and change blindness in multiple object tracking" Visual Cognition $10949-963$

Bettencourt K, Somers D, 2009 "Effects of target enhancement and distractor suppression on multiple object tracking capacity" Journal of Vision 9(7) 1 - 11

Botterill K, Allen R, McGeorge P, 2011 "Multiple-object tracking: The binding of spatial location and featural identity" Experimental Psychology 58 196-200

Brown L A, Brockmole J R, 2010 "The role of attention in binding visual features in working memory: Evidence from cognitive ageing" Quarterly Journal of Experimental Psychology $\mathbf{6 3}$ $2067-2079$

Cave K R, 1999 "The FeatureGate model of visual selection" Psychological Research 62182 - 194

Cohen M A, Pinto Y, Howe P D L, Horowitz T S, 2011 "The what - where trade-off in multiple-identity tracking" Attention, Perception, \& Psychophysics $731422-1434$

Fencsik D E, Klieger S B, Horowitz T S, 2007 "The role of location and motion information in the tracking and recovery of moving objects" Perception \& Psychophysics $69567-577$

Folk C L, Remington R, 1998 "Selectivity in distraction by irrelevant featural singletons: Evidence for two forms of attentional capture" Journal of Experimental Psychology: Human Perception and Performance $24847-858$

Folk C L, Remington R W, Johnston J C, 1992 "Involuntary covert orienting is contingent on attentional control settings" Journal of Experimental Psychology: Human Perception and Performance 181030 - 1044

Fougnie D, Marois R, 2006 "Distinct capacity limits for attention and working memory: Evidence from attentive tracking and visual working memory paradigms" Psychological Science $17526-534$

Fougnie D, Marois R, 2009 "Attentive tracking disrupts feature binding in visual working memory" Visual Cognition $1748-66$

Franconeri S L, Hollingworth A, Simons D J, 2005 "Do new objects capture attention?" Psychological Science $16275-281$

Franconeri S L, Jonathan S V, Scimeca J M, 2010 "Tracking multiple objects is limited only by object spacing, not by speed, time, or capacity" Psychological Science $21920-925$

Horowitz T S, Cohen M A, 2008 "How do distractors distract in multiple object tracking?" Perception 37 Supplement, 98 - 99 (Abstract)

Horowitz T S, Klieger S B, Fencsik D E, Yang K K, Alvarez G A, Wolfe J M, 2007 “Tracking unique objects" Perception \& Psychophysics $69172-184$

Intriligator J, Cavanagh P, 2001 "The spatial resolution of visual attention" Cognitive Psychology 43 $171-216$

Iordanescu L, Grabowecky M, Suzuki S, 2009 "Demand-based dynamic distribution of attention and monitoring of velocities during multiple-object tracking" Journal of Vision 9(4) 1 - 12

Lavie N, 1995 "Perceptual load as a necessary condition for selective attention" Journal of Experimental Psychology: Human Perception and Performance 21451 - 468

Lavie N, 2005 "Distracted and confused?: Selective attention under load" Trends in Cognitive Sciences 9 (2) $75-82$

Luck S J, Vogel E K, 1997 "The capacity of visual working memory for features and conjunctions" Nature $390279-281$ 
Makovski T, Jiang Y, 2009a "Feature binding in attentive tracking of distinct objects" Visual Cognition $17180-194$

Makovski T, Jiang Y, 2009b "The role of visual working memory in attentive tracking of unique objects" Journal of Experimental Psychology: Human Perception \& Performance 351687 - 1697

Oksama L, Hyönä J, 2004 "Is multiple object tracking carried out automatically by an early vision mechanism independent of higher-order cognition? An individual difference approach" Visual Cognition $11631-671$

Oksama L, Hyönä J, 2008 "Dynamic binding of identity and location information: A serial model of multiple identity tracking" Cognitive Psychology $56237-283$

Pinto Y, Olivers C N L, Theeuwes J, 2006 "When is search for a static target among dynamic distractors efficient?" Journal of Experimental Psychology: Human Perception and Performance $3259-72$

Pylyshyn Z W, 2004 "Some puzzling findings in multiple object tracking (MOT): I. Tracking without keeping track of object identities" Visual Cognition 11 $801-822$

Pylyshyn Z W, 2006 "Some puzzling findings in multiple object tracking (MOT): II. Inhibition of moving nontargets" Visual Cognition $14175-198$

Pylyshyn Z, Haladjian H, King C, Reilly J, 2008 "Selective nontarget inhibition in multiple object tracking (MOT)" Visual Cognition $161011-1021$

Pylyshyn Z W, Storm R W, 1988 "Tracking multiple independent targets: Evidence for a parallel tracking mechanism" Spatial Vision $3179-197$

Quinlan P T, Humphreys G W, 1987 "Visual search for targets defined by combinations of color, shape, and size: An examination of the task constraints on feature and conjunction searches" Perception \& Psychophysics $41455-472$

Ren D, Chen W, Liu C H, Fu X, 2009 "Identity processing in multiple-face tracking" Journal of Vision 9(5) $1-15$

Saiki J, 2003 "Spatiotemporal characteristics of dynamic feature binding in visual working memory" Vision Research 43 2107-2123

Saiki J, Miyatsuji H, 2007 "Feature binding in visual working memory evaluated by type identification paradigm" Cognition $10249-83$

Sears C R, Pylyshyn Z W, 2000 "Multiple object tracking and attentional processing" Canadian Journal of Experimental Psychology 541 - 14

Shim W M, Alvarez G A, Jiang Y V, 2008 "Spatial separation between targets constrains maintenance of attention on multiple objects" Psychonomic Bulletin \& Review $15390-397$

Stanislaw H, Todorov N, 1999 "Calculation of signal detection theory measures" Behavior Research Methods, Instruments, \& Computers 31 137-149

St Clair R, Huff M, Seiffert A E, 2010 "Conflicting motion information impairs multiple object tracking" Journal of Vision 10(4) $1-13$

Störmer V S, Li S-C, Heekeren H R, Lindenberger U, 2011 "Feature-based interference from unattended visual field during attentional tracking in younger and older adults" Journal of Vision 11(2) $1-12$

Theeuwes J, 1992 "Perceptual selectivity for color and form" Perception \& Psychophysics 51599 - 606

Theeuwes J, 1994 "Stimulus-driven capture and attentional set: Selective search for color and visual abrupt onsets" Journal of Experimental Psychology: Human Perception and Performance 20 799 - 806

Treisman A M, Gelade G, 1980 "A feature-integration theory of attention" Cognitive Psychology $1297-136$

Treisman A, Sato S, 1990 "Conjunction search revisited" Journal of Experimental Psychology: Human Perception and Performance 16459 - 478

Viswanathan L, Mingolla E, 2002 "Dynamics of attention in depth: Evidence from multi-element tracking" Perception $311415-1437$

Wolfe J M, 1994 "Guided Search 2.0: A revised model of visual search" Psychonomic Bulletin \& Review $1202-238$

Wolfe J M, Cave K R, Franzel S L, 1989 "Guided search: An alternative to the feature integration model for visual search" Journal of Experimental Psychology: Human Perception and Performance 15419 - 433

Yantis S, 1992 "Multielement visual tracking: Attention and perceptual organization" Cognitive Psychology 24295 - 340 


\section{PERCEPTION}

VOLUME 412012

www.perceptionweb.com

Conditions of use. This article may be downloaded from the Perception website for personal research by members of subscribing organisations. Authors are entitled to distribute their own article (in printed form or by e-mail) to up to 50 people. This PDF may not be placed on any website (or other online distribution system) without permission of the publisher. 